OPEN ACCESS

Edited by:

Oscar Escribano,

Complutense University of

Madrid, Spain

Reviewed by:

Carmine Finelli,

ASL Napoli 3 Sud, Italy

Giuseppe Calamita,

University of Bari Aldo Moro, Italy

${ }^{*}$ Correspondence:

Mark D. Muthiah

mdcmdm@nus.edu.sg orcid.org/0000-0002-9724-4743

Cheng Han Ng

chenhanng@gmail.com orcid.org/0000-0002-8297-1569

Specialty section: This article was submitted to Gastroenterology,

a section of the journal

Frontiers in Medicine

Received: 06 April 2021 Accepted: 26 May 2021

Published: 30 June 2021

Citation:

Xiao J, Lim LKE, Ng CH, Tan DJH, Lim WH, Ho CSH, Tan EXX, Sanyal AJ and Muthiah MD (2021) Is Fatty Liver

Associated With Depression? A Meta-Analysis and Systematic Review on the Prevalence, Risk Factors, and

Outcomes of Depression and Non-alcoholic Fatty Liver Disease. Front. Med. 8:691696. doi: 10.3389/fmed.2021.691696

\section{Is Fatty Liver Associated With Depression? A Meta-Analysis and Systematic Review on the Prevalence, Risk Factors, and Outcomes of Depression and Non-alcoholic Fatty Liver Disease}

Jieling Xiao ${ }^{1}$, Lincoln Kai En Lim ${ }^{1}$, Cheng Han Ng ${ }^{1 *}$, Darren Jun Hao Tan ${ }^{1}$, Wen Hui Lim ${ }^{1}$, Cyrus S. H. Ho ${ }^{1,2}$, Eunice Xiang Xuan Tan ${ }^{1,3,4}$, Arun J. Sanyal ${ }^{5}$ and Mark D. Muthiah ${ }^{1,3,4 *}$

${ }^{1}$ Yong Loo Lin School of Medicine, National University of Singapore, Singapore, Singapore, ${ }^{2}$ Department of Psychological Medicine, Yong Loo Lin School of Medicine, National University of Singapore, Singapore, Singapore, ${ }^{3}$ National University Centre for Organ Transplantation, National University Health System, Singapore, Singapore, ${ }^{4}$ Division of Gastroenterology and Hepatology, Department of Medicine, National University Hospital, Singapore, Singapore, ${ }^{5}$ Division of Gastroenterology, Hepatology, and Nutrition, Department of Internal Medicine, Virginia Commonwealth University, Richmond, VA, United States

Background and Aims: Both non-alcoholic fatty liver disease (NAFLD) and depression have a high global prevalence which is projected to increase further. While studies exploring the association have been done, there are conflicting data. This study aims to assess the prevalence and association between depression and NAFLD.

Methods: Medline and Embase were searched from inception to March 3, 2020. Meta-analysis of proportions using the generalized linear mix model was conducted to analyze the pooled prevalence of depression in NAFLD patients. Risk factors for depression in NAFLD patients were evaluated in conventional pairwise meta-analysis.

Results: Ten studies involving 2,041,752 NAFLD patients were included. Pooled prevalence of depression was 18.21\% (Cl: 11.12-28.38\%) in patients with NAFLD and $40.68 \%$ (Cl: $25.11-58.37 \%)$ in patients with non-alcoholic steatohepatitis (NASH). NAFLD resulted in significantly higher risk of development of depression (OR: 1.29, Cl: 1.02-1.64, $p=0.03$ ). NASH patients had a significantly higher risk of depression compared with NAFLD patients (RR: 2.83, Cl: 2.41-3.32, $p<0.001$ ). Diabetes, body mass index (BMI), female sex, smoking, and history of pulmonary disease were significant risk factors for depression in NAFLD patients.

Conclusion: This study demonstrated a high prevalence of depression in NAFLD patients and a significant association between both conditions. Furthermore, patients with NASH had a significantly higher risk of depression compared with those with NAFLD. Diabetes, BMI, history of lung disease or smoking, and female gender were significant risk factors. Further studies investigating the pathophysiological mechanism underlying depression and NAFLD are needed.

Keywords: mood disorder, metabolic disease, fatty liver, evidence-based practice, mental health 


\section{INTRODUCTION}

Non-alcoholic fatty liver disease (NAFLD) is the most common cause of chronic liver disease, with an estimated global prevalence of $25 \%$. This is further projected to increase in conjunction with the rising rates of metabolic syndrome and obesity (1-4). However, the influence of depression on NAFLD has yet to be well-described. The Global Burden of Disease study from 1990 to 2017 reported a $49.86 \%$ increase in the incidence of depression (5), with at least one in five people experiencing depression in their lifetime (6-8). Depression is often comorbid of many chronic diseases and incrementally worsens health outcomes $(9,10)$. Multiple community- and population-based studies have reported that patients with depression have a 2 -fold increased risk of developing metabolic syndrome (11-13). Depression is also highly prevalent in the diabetic population, affecting more than one-quarter of both type 1 and type 2 diabetics (14). A metaanalysis of longitudinal studies also confirmed the reciprocal relationship between obesity and depression (15) and increased cardiac mortality in patients with this comorbidity (16).

While studies have been conducted to describe the relationship between depression and NAFLD, previous literature has reported conflicting results, varying from a strong association $(17,18)$ to no association $(19,20)$. A study involving a database of 567 patients with biopsy-proven NAFLD estimated that $67.5 \%$ of patients had depressive symptoms and showed that they were associated with histological severity of NAFLD (21). It is unclear if depression affects NAFLD due to underlying risk factors or if depression is independently associated with NAFLD (22). Thus, this meta-analysis and systematic review aims to assess the prevalence, associations, risk factors, and outcomes between depression and NAFLD.

\section{METHODS}

\section{Search Strategy}

This review was synthesized with reference to the Preferred Reporting Items for Systematic Reviews and Meta-Analyses (PRSIMA) guidelines (23). A search was conducted on Medline and Embase databases to identify relevant articles from inception up to March 3, 2020. Keywords and MeSH terms synonymous to "NAFLD" and "depression" were applied in the search strategy to identify relevant articles. The full search used was as follows: (depress*.tw. or exp Depression/or MDD.tw.) AND (NAFLD or NASH or ((liver or hepatic) AND (fatty or steatosis or steatoses))).tw. or (exp Fatty Liver/or steatohepat*tw.). In addition, a sieve was conducted on the references of included articles. Abstracts were imported into EndNote X9 for removal of duplicates and for the initial sieve.

\footnotetext{
Abbreviations: BMI, body mass index; CES-D, Korean Center for Epidemiological Studies-Depression Scale; COPD, chronic obstructive pulmonary disease; DSMIV-TR, Diagnostic and Statistical Manual of Mental Disorders, 4th edition, text revision; HADS, Hospital Anxiety and Depression Scale; ICD-9 and ICD10, International Classification of Disease, Ninth Revision and Tenth Revision; NAFLD, non-alcoholic fatty liver disease; NASH, non-alcoholic steatohepatitis; MDD, major depressive disorder; PHQ-9, Patient Health Questionnaire-9.
}

\section{Study Selection and Data Extraction}

Two authors (XJL and LLKE) were involved in the screening of abstracts to check the eligibility for inclusion, with disputes being resolved through consensus from a third independent author. Retrospective and prospective cohort studies, case-control, and cross-sectional studies were considered for inclusion, while editorials, systematic reviews, meta-analyses, and commentaries were excluded. Additionally, only English language articles were considered for inclusion. Studies were included according to the following criteria: i) studies regarding the prevalence, risk factors, and outcomes of depression and ii) studies where patients had a diagnosis of NAFLD made via liver biopsy, imaging techniques (radiologic testing and abdominal ultrasound), or International Classification of Diseases. Studies relating to the diagnosis of non-alcoholic steatohepatitis (NASH) by selfreported physician diagnosis were also included. Since NASH is a subgroup of NAFLD, a definitive diagnosis of NASH would equate to the presence of NAFLD. As with a previous article, the diagnosis of depression was subclassified into self-reported, selfrated, and clinician-rated (24). Self-reported diagnosis includes identification of depression through self-reporting of medical history, while self-rated diagnosis of depression comprises of patient responses from the Patient Health Questionnaire9 (PHQ-9), Hospital Anxiety and Depression Scale (HADS), Korean Center for Epidemiological Studies-Depression Scale (CES-D), and Beck's Depression Inventory scale. Clinician-rated diagnosis comprises of depression diagnosed by a psychiatrist according to the criteria of the Diagnostic and Statistical Manual of Mental Disorders, 4th edition, text revision (DSM-IV-TR) and International Classification of Disease, Ninth Revision (ICD9) and Tenth Revision (ICD-10) codes. Pediatrics studies were excluded from the analysis.

Relevant data from each article were extracted by a pair of independent authors onto a structured proforma. Baseline demographics, including but not limited to author, year, sample size, country, mean age, percentage of diabetes, and prevalence of depression, were extracted. The main outcomes of interest were the prevalence of depression in NAFLD and NASH patients and risk factors including gender, diabetes mellitus, body mass index (BMI), hypertension, hyperlipidemia, history of smoking, history of lung disease, history of cancer, history of heart diseases, history of neurologic diseases, and race.

\section{Statistical Analysis and Quality Assessment}

All analysis was done in R studio (Version 1.3.1093) and RevMan 5.4. Statistical significance was considered for outcomes with a $p$-value $<0.05$. A single-arm analysis of binary outcomes was pooled in the form of proportions using the generalized linear mixed model (GLMM) with Clopper-Pearson intervals to stabilize the variance $(25,26)$. Simulation studies have found that the GLLM model provides the most accurate estimate in single-arm meta-analysis (25). A sensitivity analysis was done to include only studies from biopsy-proven NAFLD. Next, a subgroup analysis was conducted to account for the differences in the rate of depression between clinician-diagnosed, self-reported, 
and self-rated diagnosis. Based on the pooled proportions of single-arm studies, the respective relative risks (RR) of depression in NASH $\left(p_{1}\right)$ vs. NAFLD $\left(p_{2}\right)$ patients was calculated as the ratio of the pooled proportions $\frac{p_{1}}{p_{2}}$ of patients with depression in each subgroup (27). The lower (LCL) and upper (UCL) bounds for the $95 \%$ confidence intervals were estimated using the Katz-logarithmic method, wherein $n_{1}$ and $n_{2}$ represent the respective number of patients receiving transplanted allografts fixated using each of the three methods. Additionally, the $p$-value was calculated after a natural log transformation of the relative risk $z$-score (28).

$$
\begin{gathered}
L C L=R R e^{\left(-1.96 \times \sqrt{\frac{1-p_{1}}{n_{1} p_{1}}+\frac{1-p_{2}}{n_{2} p_{2}}}\right)} \\
U C L=R R e^{\left(1.96 \times \sqrt{\frac{1-p_{1}}{n_{1} p_{1}}+\frac{1-p_{2}}{n_{2} p_{2}}}\right)} \\
p-\text { value }=e^{\left(-0.717 \times\left|\frac{\ln R R}{\frac{\ln U C L-\ln L C L}{2 \times 1.96}}\right|-0.416 \times\left(\frac{\ln R R}{\frac{\ln U C L-\ln L C L}{2 \times 1.96}}\right)^{2}\right)}
\end{gathered}
$$

In the analysis of risk factors, a conventional pairwise analysis was done using odds ratios (OR) and mean difference (MD) with the Mantel-Haenszel and inverse variance, respectively $(29,30)$. For outcomes with insufficient articles for a metaanalysis, a systematic synthesis of literature was used to represent available data. Statistical heterogeneity was assessed via $I^{2}$ and Cochran $Q$ test values, where an $I^{2}$ value of 0 to $40 \%$ indicates low heterogeneity, while values of $30-60,50-90$, and 75$100 \%$ were classified as moderate, substantial, and considerable heterogeneity, respectively $(29,31)$. A Cochran $Q$ test with $p$-value of $\leq 0.10$ was considered significant for heterogeneity. A random effects model was used in all analysis regardless of heterogeneity as recent evidence suggests that it provides more robust outcome measures compared with the alternative fixed effects models. Publication bias was not assessed with the lack of a suitable tool in single-arm meta-analysis to assess publication bias and small quantity of included studies $(32,33)$. Quality assessment of the included articles was done with the Joanna Briggs Institute (JBI) Critical Appraisal Tool (34). The JBI assessment rates the risk of bias of cohort studies on the premises of appropriateness of sample frame, sampling method, adequacy of sample size, data analysis, methods for identification and measurement of relevant conditions, statistical analysis, and response rate adequacy and is the most widely used tool in prevalence meta-analysis.

\section{RESULTS}

\section{Summary of Included Articles}

In the initial search strategy, 1,766 references were identified, of which 1,315 references were screened after the removal of duplicates. After initial screening, a full-text review was done for 50 articles, and finally, 10 articles involving 2,041,752 NAFLD patients were included in the analysis (Figure 1). Of the included studies, one is a prospective study (35), while the remainder are retrospective studies $(17,21,22,36-41)$. Depression was identified through clinician-rated scales [National Institute of Mental Health Diagnostic Interview Schedule (DIS), Version III (36); DSM-IV-TR (35) and International Classification of Disease, Ninth Revision (ICD-9) and Tenth Revision (ICD10) $(41,42)]$, self-rated scales [HADS (21), CES-D (38), Beck Depression Inventory scale (22)], and self-reported physician diagnosis $(17,37,40)$. NAFLD was diagnosed through liver biopsy in four studies $(17,21,35,40)$ and imaging techniques in four studies $(17,22,38,40)$ and identified through ICD-9 and ICD-10 codes in two studies $(41,42)$. NASH was diagnosed through liver biopsy in one study (36) and identified through selfreported physician diagnosis in another study (37). A summary of the included articles and quality assessment can be found in Supplementary Tables 1, 2, respectively.

\section{Development of Depression in NAFLD}

Five studies assessed the association of depression with NAFLD $(21,22,36,38,42)$. In the pooled analysis of the four retrospective studies involving 38,407 patients, NAFLD resulted in a significant increase in the risk of depression (OR: 1.29, 95\% CI: $1.02-1.64, p=0.03$, Figure 2). A retrospective followup study by Labenz et al. assessed the incidence of depression in 19,871 NAFLD patients over 10 years. The 10 -year incidence of depression was $21.2 \%$ in patients with NAFLD compared with $18.2 \%$ of individuals without NAFLD. The hazard ratio was 1.21 (95\% CI: $1.14-1.26, p<0.001)$ risk increase in development depression in NALFD patients after adjusting for confounders including diabetes mellitus, cardiovascular diseases, asthma, chronic obstructive pulmonary disease (COPD), and cancer (42).

\section{Prevalence of Depression in NAFLD}

The overall pooled prevalence of depression in patients with NAFLD was $18.21 \%$ (CI: 11.12-28.38\%, Figure 3) in 2,041,752 individuals. The pooled prevalence of depression in biopsyproven NAFLD was $22.68 \%$ (CI: $8.93-46.75 \%$ ).

A subgroup analysis was conducted to compare between NASH and NAFLD. The prevalence of depression in NASH patients is $40.68 \%$ (CI: $25.11-58.37 \%$ ), significantly higher than the pooled prevalence of depression in NAFLD patients at 14.39\% (CI: 8.89-22.45\%). Compared with patients with NAFLD, patients with NASH had a significantly higher risk of depression (RR: 2.83, CI: 2.41-3.32, $p<0.001$ ).

To account for differences in the diagnosis of depression as a potential source for heterogeneity, estimates were stratified by diagnostic criteria of depression. Prevalence of depression in NAFLD patients in four studies where depression was identified through clinician-rated scales was $20.26 \%$ (CI: 9.38-38.42\%) (35, $36,41,42)$, which was lower than self-reported diagnosis used in three studies where prevalence was $32.43 \%$ (CI: 27.03-38.33\%) $(17,37,40)$ and higher than self-rated depression reported in three studies where prevalence was $8.19 \%$ (CI: $4.66-14.01 \%$ ) $(21,22,38)$. 

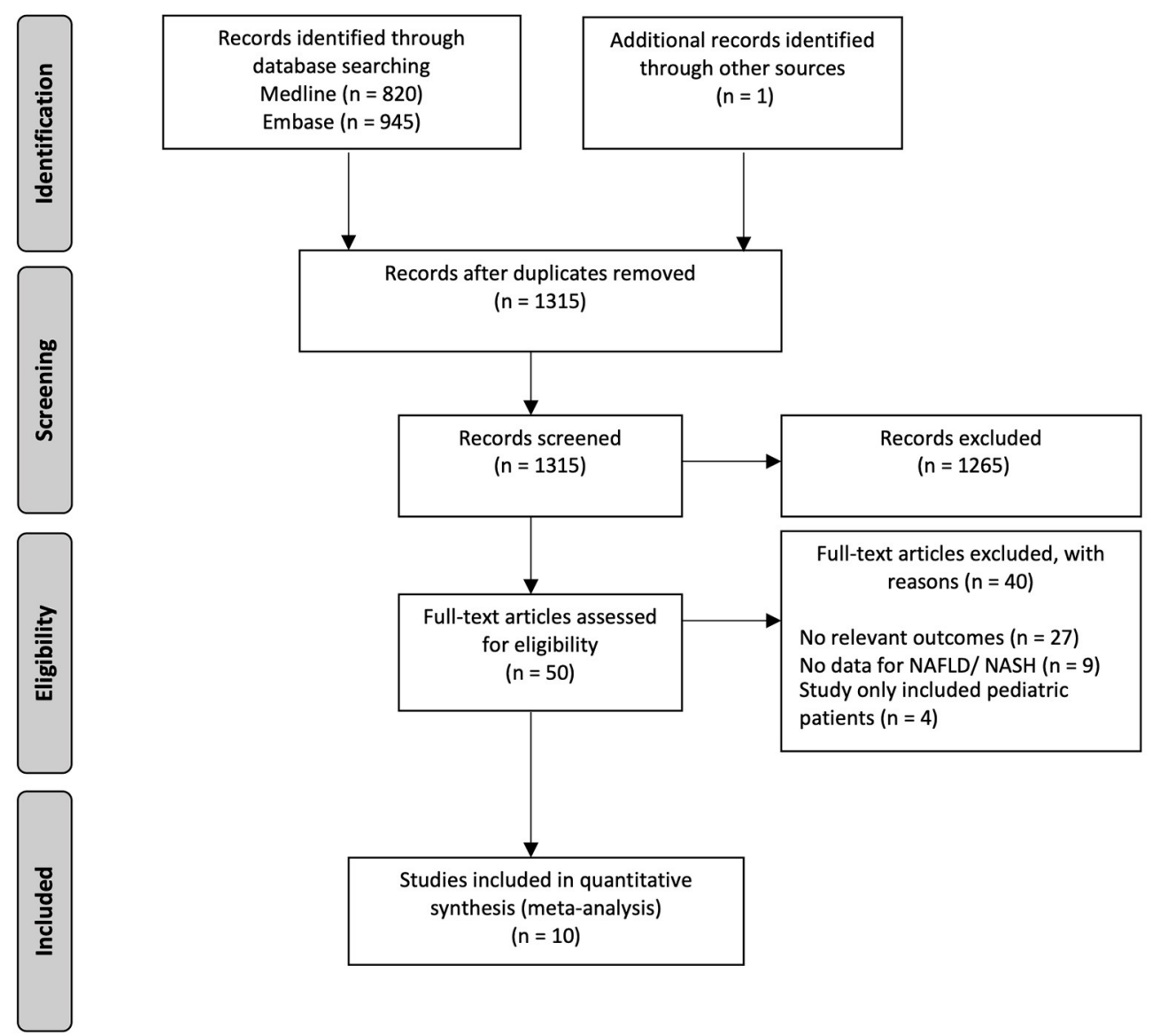

FIGURE 1 | PRISMA flowchart of the included studies.

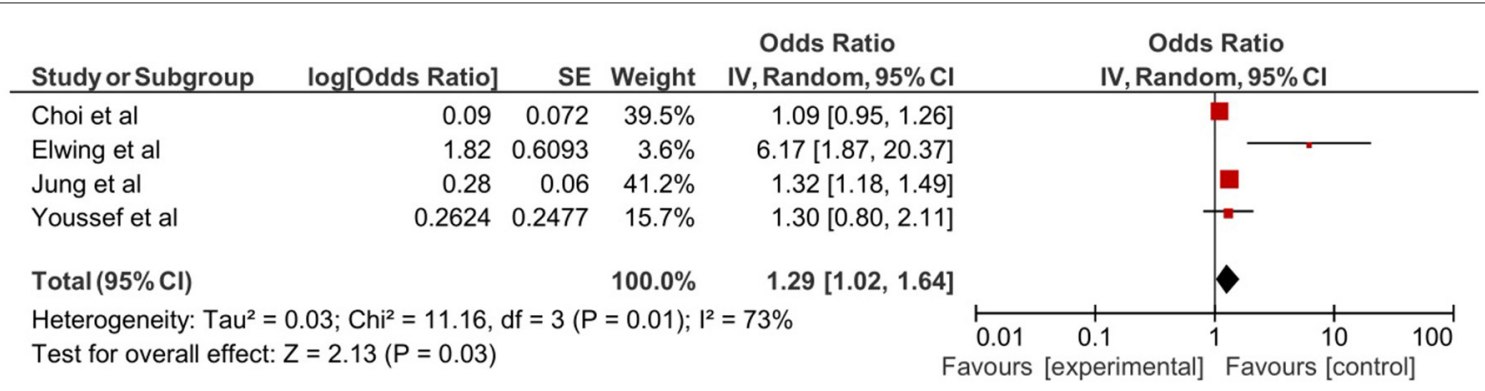

FIGURE 2 | Forest plot of depression in non-alcoholic fatty liver disease (NAFLD).

\section{Factors Associated With Depression in NAFLD}

When comparisons were made between NAFLD patients with depression and NAFLD patients without depression, diabetes (OR: 1.71, CI: 1.15-2.55, $p=0.007$ ), BMI (MD: 1.89, CI: 0.972.80, $p<0.0001$ ), and female sex (OR: 0.57, CI: 0.35-0.93, $p=0.02$ ) were significant risk factors associated with depression in NAFLD patients. However, hypertension (OR: 1.54, CI: 1.002.37, $p=0.05$ ) and hyperlipidemia (OR: 1.18, CI: 0.61-2.28, $p=0.623)$ were not significant risk factors for depression in NAFLD patients.

In addition, Weinstein et al. (17) reported history of smoking and history of lung disease as statistically significant risk factors independently associated with depressions in NAFLD patients (OR: 4.132, CI: $1.224-13.95, p=0.0096$ and OR: 4.621, CI: $1.346-$ 15.92, $p=0.0087$, respectively). A previous history of cancer (OR: 1.370, CI: 0.394-4.765, $p=0.621$ ), heart diseases (OR: 2.750, CI: $0.377-20.07, p=0.319$ ), or neurologic disease (OR: 


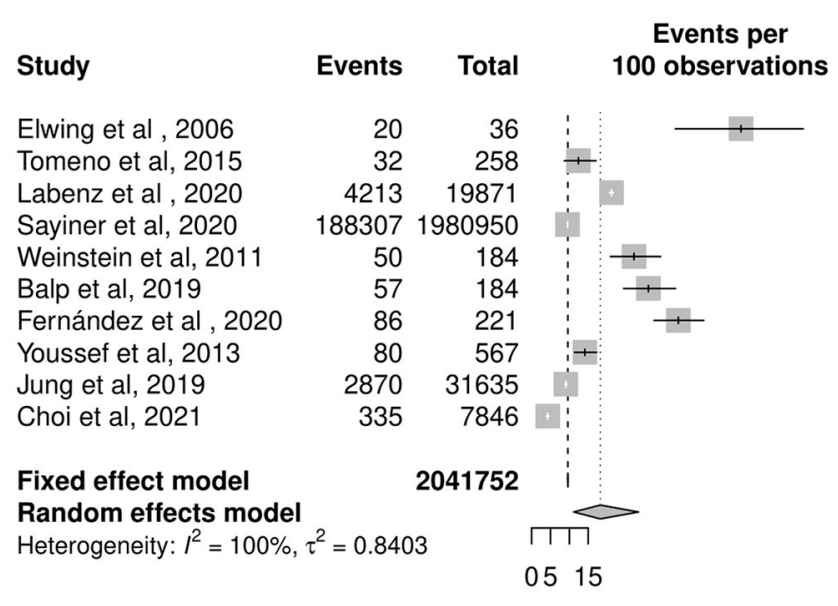

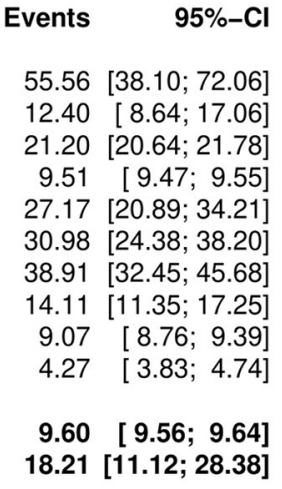

18.21 [11.12; 28.38]

FIGURE 3 | Pooled prevalence of depression in NAFLD patients.

2.787, CI: $0.544-14.29, p=0.219$ ) had no significant association with depression. African American descent was not associated with the development of depression (OR: 0.198, CI: 0.024$1.629, p=0.147)$.

\section{Outcomes of NAFLD With Depression}

Two studies, Tomeno et al. and Sayiner et al. reported on the outcomes of NAFLD patients comorbid with depression. Tomeno et al. compared the clinical response of 32 NAFLD patients with major depressive disorder (MDD) and 226 NAFLD patients without MDD after 48 weeks of standard care involving basic education, lifestyle change counseling, and medication control. Parameters used in the calculation of hepatic steatosis index and fatty liver index such as body weight, serum AST, ALT, and GGT levels were significantly improved in NAFLD patients without MDD after 48 weeks of standard care. In contrast, there were no statistically significant improvements in these parameters for NAFLD patients with MDD (35). Sayiner et al. analyzed depression as an independent predictor of overall 1-year mortality in NAFLD patients in both inpatient and outpatient settings. The odds for overall 1-year mortality of NAFLD patients with depression was statistically significant in both inpatient (OR: 1.07, CI: 1.05-1.09) and outpatient settings (OR: 1.21, CI: $1.18-1.25)(41)$.

\section{DISCUSSION}

The projected rise in the incidence of NAFLD mirrors the obesity epidemic, which at present affects at least one-third of the world (43). This systematic review and meta-analysis reports $15.76 \%$ prevalence of depression in NAFLD patients. The risk of development of depression was significantly increased in patients with NAFLD (OR: 1.29, 95\% CI: 1.02-1.64, $p=0.03$ ). A 10-year follow-up by Labenz et al. also showed a significant association between depression and NAFLD (HR: 1.21, 95\% CI: 1.14-1.26, $p<0.001)$. Female sex, diabetes, BMI, history of smoking, and history of lung disease were associated with the development of depression. The relative risk of depression between NASH and NAFLD patients was RR: 2.83 (CI: 2.41-3.32, $p<0.001$, Figure 4). In truth, the actual prevalence of clinically diagnosed depression is likely to be higher than that reported, especially among Asians where traditional screening methods for depression have been reported to have a poorer detection rate (44).

The incidence of depression is likely to be higher in patients with NAFLD as compared with those without, as shown by Labenz et al. in their large population-based study (42). Importantly, the authors controlled for variables including diabetes and obesity, demonstrating increased incidence of depression in patients with NAFLD independent of these comorbidities. While the studies included in our manuscript are unable to show causality, there is emerging evidence, which may shed light on the associations between depression and NAFLD. Systemic inflammation plays a putative role in the pathogenesis of NAFLD and depression, and the progression of both diseases is seen in states of increased oxidative stress (45). Increased levels of proinflammatory cytokines such as interleukin-6, interleukin-1 beta, and tumor necrosis factor alpha may contribute to systemic inflammation leading to depression in NAFLD patients $(42,46,47)$. Another plausible association between NAFLD and depression would be the role of serotonin in the pathogenesis of depression as there is increased expression of serotonin catalyzing enzymes in patients with NASH (22).

In our study, diabetes was a significant risk factor for the development of depression (OR: 1.71, CI: 1.15-2.55, $p=0.007$ ). Egede et al. reported diabetic patients to be almost twice as likely to be diagnosed with depression compared with the general population (48). BMI was identified as a significant risk factor for the development of depression (MD: 1.89, CI: 0.97-2.80, $p<0.0001$ ). There are also several studies reporting an increased prevalence of depression with higher BMI $(49,50)$. Luppino et al. in their meta-analysis of obesity and depression showed that obesity at baseline had increased the risk of onset of depression at follow-up, with OR 1.55 (95\% CI 1.22-1.98, $p<0.001$ ) (15). 


\section{NAFLD/NASH and Depression}

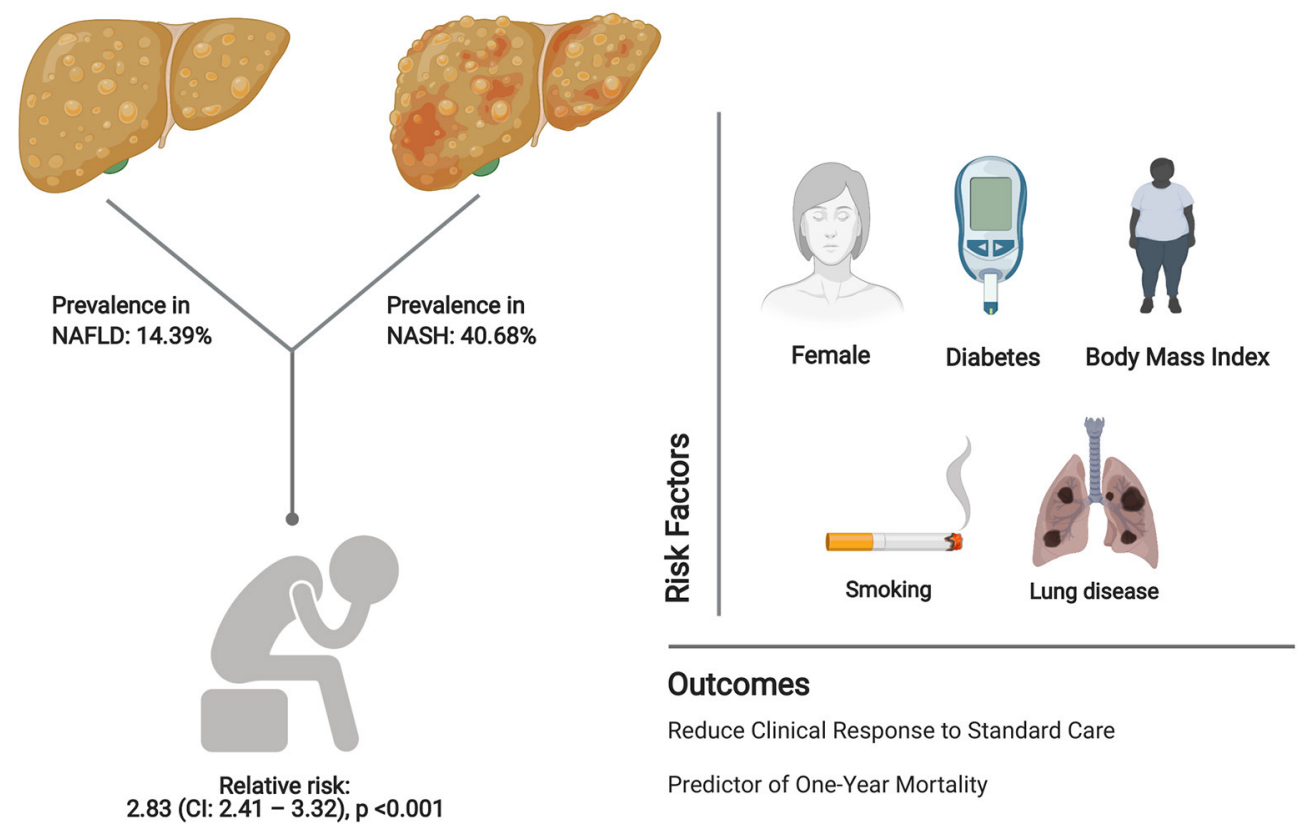

FIGURE 4 | Summary of prevalence, risk factors, and clinical outcomes of depression in NAFLD patients. Image created with Biorender.com.

The risk factors of depression in NAFLD patients were associated with increased systemic inflammation as observed in smokers and patients with chronic lung disease. Female gender was also associated with increased odds of depression in NAFLD patients. It is known that females are at higher risk of depression than males, and a study in 2015 by Moieni suggested that during an induced state of inflammation, women encountered greater increases in depression (51). Smoking has also been identified as a risk factor for depression, possibly due to the role of nicotine receptors as neuromodulators of various neurotransmitter pathways to the brain, including those involved in depression (52). This in turn may have contributed to history of lung disease being a risk factor for depression as corroborated by Goodwin et al. (53).

Interestingly, the prevalence of depression is markedly higher in patients with NASH as compared with patients with simple hepatic steatosis. NASH with or without fibrosis is a more severe disease compared with simple steatosis. It occurs at a rate of about $25 \%$ in 3 years in patients in the simple steatosis stage (54). This would not come as a surprise given that an increase in circulating inflammatory cytokines is seen in steatohepatitis compared with simple steatosis, and patients with depression similarly see an increase in peripheral and central inflammation. In fact, the bidirectional relationship between depression and obesity, which is also closely related to NAFLD, has already been elucidated in animal studies (55). Therefore, it is likely that dietary initiatives protective toward NAFLD progression may also improve mental health in patients with depression (56). Evidently, depression and NAFLD share multiple upstream hits in the pathophysiology of the disease; thus, it is no wonder that they are gradiently associated.

In a study by Tomeno et al. the presence of depression was associated with a decreased effect of lifestyle intervention on weight loss, resulting in an increased challenge for clinical management (35). Although there are multiple pharmacotherapies with promising results in phase 3 trials for $\mathrm{NASH}$, at present, weight loss and lifestyle change are the cornerstones of NAFLD treatment. Furthermore, overall weight loss has been shown to be beneficial for associated comorbidities such as diabetes and hypertension $(57,58)$. Yet, many antidepressant classes result in weight gain, and thus, the possibility of successful treatment outcome may be grim in the coexistence of depression and NAFLD (59).

It may be tempting to consider bariatric surgery for this subgroup of patients with both NAFLD and depression. After all, bariatric surgery has shown promising results in improvement in NASH as seen in several studies (60-62). Furthermore, weight loss following bariatric surgery may be associated with improvements in mood, at least initially. However, it is not without adverse outcomes. For example, a 10-year cohort study in Sweden reported an increase in suicides among obese patients following surgery compared with the general population (OR: 2.85, 95\% CI: 2.40-3.39) in addition to alcohol and substance abuse (63). Several reasons for increased suicide rates post-bariatric surgery have been suggested, such as genetic susceptibility and changes in gut peptide release. It has also been theorized that addiction transfer could explain this phenomenon. In this theory, binge eating is hypothesized to 
serve as a coping mechanism to ameliorate negative emotional states (64). Consequently, in the post-bariatric group, this behavior is substituted by alcohol consumption due to the patient's inability to regulate emotions through overeating. However, this model has neither been adequately developed nor empirically validated and should be explored in future research (65).

Depression in NAFLD is associated with poorer outcomes such as decreased response to medical treatment and is even an independent predictor of all-cause mortality at 1 year. It is unclear if treatment of depression (be it pharmacological or behavioral) would positively influence NAFLD progression. Nevertheless, physicians should be cognizant of the fact that the prevalence and incidence of depression in NAFLD patients is higher and may consider adopting a low threshold in the use of screening scores for mood disorders in these patients as clinically required.

\section{Strengths and Limitations}

To our best knowledge, this is the first meta-analysis of the prevalence, associative risk factors, and outcomes of depression in NAFLD patients. The limitation of this study is the heterogeneity in the included studies. However, we circumvented the issue by reclassification of depression diagnosis into clinicianrated, self-reported, and self-rated scales (66). Of note, the prevalence of depression was higher in self-reported and selfrated studies. Moreover, larger sample sizes are often associated with an increased $I^{2}$ in simulation studies $(67,68)$. Thus, large $I^{2}$ value $(>90 \%)$ in prevalence meta-analysis may be attributed to the larger sample sizes involved $(69,70)$. Secondly, while the prevalence of NAFLD in Asia is similar to the Western population, only three out of the 10 studies were conducted in Asia. Thus, this meta-analysis may not adequately represent Asian NAFLD patients. Lastly, while our study reports factors associated with depression in NAFLD patients, more confirmatory studies are needed to show causation through further understanding of the pathogenesis and bidirectional relationship between the two conditions.

\section{REFERENCES}

1. Neuschwander-Tetri BA. Non-alcoholic fatty liver disease. BMC Med. (2017) 15:45. doi: 10.1186/s12916-017-0806-8

2. Rinella ME. Nonalcoholic fatty liver disease: a systematic review. JAMA. (2015) 313:2263-73. doi: 10.1001/jama.2015.5370

3. Finelli C, Tarantino G. What is the role of adiponectin in obesity related non-alcoholic fatty liver disease? World J Gastroenterol. (2013) 19:802-12. doi: 10.3748/wjg.v19.i6.802

4. Cho IY, Chang Y, Sung E, Kang JH, Wild SH, Byrne CD, et al. Depression and increased risk of non-alcoholic fatty liver disease in individuals with obesity. Epidemiol Psychiatr Sci. (2021) 30:e23. doi: 10.1017/S20457960200 0116X

5. Liu Q, He H, Yang J, Feng X, Zhao F, Lyu J. Changes in the global burden of depression from 1990 to 2017: findings from the global burden of disease study. J Psychiatr Res. (2020) 126:134-40. doi: 10.1016/j.jpsychires.2019.08.002

6. Malhi GS, Mann JJ. Depression. Lancet. (2018) 392:2299-312. doi: 10.1016/S0140-6736(18)31948-2

\section{CONCLUSION}

In summary, this meta-analysis and systematic review assesses the association between depression and NAFLD. The analysis demonstrates the high prevalence of depression in NAFLD in spite of heterogeneity due to differences in diagnostic criteria of depression. Furthermore, patients with NASH were found to have a significantly higher risk of depression compared with those with NAFLD. Diabetes, BMI, history of smoking, history of lung disease, and being female were also identified as significant risk factors. NAFLD comorbid with depression has serious complications as evident in reduced clinical response after standard care and increased all-cause 1-year mortality. This paper, thus, highlights the significant clinical implications of NAFLD and depression as public health concerns. Further studies to validate the findings and explore potential pathophysiological mechanism underlying the association between depression and NAFLD are needed.

\section{DATA AVAILABILITY STATEMENT}

The original contributions presented in the study are included in the article/Supplementary Material, further inquiries can be directed to the corresponding authors.

\section{AUTHOR CONTRIBUTIONS}

JX, LL, and CN contributed to the acquisition of data, analysis and interpretation of data, and drafting of the article. DT, WL, $\mathrm{CH}, \mathrm{ET}, \mathrm{AS}$, and $\mathrm{MM}$ aided in revising the article critically for important intellectual content. All authors read and gave final approval of the version to be submitted.

\section{SUPPLEMENTARY MATERIAL}

The Supplementary Material for this article can be found online at: https://www.frontiersin.org/articles/10.3389/fmed. 2021.691696/full\#supplementary-material

7. Herrman H, Kieling C, McGorry P, Horton R, Sargent J, Patel V. Reducing the global burden of depression: a lancet-World psychiatric association commission. Lancet. (2019) 393:e42-3. doi: 10.1016/S0140-6736(18)32408-5

8. Patel V, Chisholm D, Parikh R, Charlson FJ, Degenhardt L, Dua T, et al. Addressing the burden of mental, neurological, and substance use disorders: key messages from disease control priorities, 3rd edition. Lancet. (2016) 387:1672-85. doi: 10.1016/S0140-6736(15)00390-6

9. Moussavi S, Chatterji S, Verdes E, Tandon A, Patel V, Ustun B. Depression, chronic diseases, and decrements in health: results from the world health surveys. Lancet. (2007) 370:851-8. doi: 10.1016/S0140-6736(07)61415-9

10. Gawlik-Kotelnicka O, Strzelecki D. Adiposity in depression or depression in adiposity? The role of immune-inflammatory-microbial overlap. Life. (2021) 11:117. doi: 10.3390/life11020117

11. Goldbacher EM, Bromberger J, Matthews KA. Lifetime history of major depression predicts the development of the metabolic syndrome in middle-aged women. Psychosom Med. (2009) 71:266-72. doi: 10.1097/PSY.0b013e318197a4d5

12. Roriz-Cruz M, Rosset I, Wada T, Sakagami T, Ishine M, Roriz-Filho JS, et al. Stroke-independent association between metabolic syndrome and 
functional dependence, depression, and low quality of life in elderly community-dwelling Brazilian people. J Am Geriatr Soc. (2007) 55:374-82. doi: 10.1111/j.1532-5415.2007.01068.x

13. Kinder LS, Carnethon MR, Palaniappan LP, King AC, Fortmann SP. Depression and the metabolic syndrome in young adults: findings from the third national health and nutrition examination survey. Psychosom Med. (2004) 66:316-22. doi: 10.1097/01.psy.0000124755.91880.f4

14. Lustman PJ, Clouse RE. Depression in diabetic patients: the relationship between mood and glycemic control. J Diabetes Complicat. (2005) 19:113-22. doi: 10.1016/S1056-8727(04)00004-2

15. Luppino FS, de Wit LM, Bouvy PF, Stijnen T, Cuijpers P, Penninx BW, et al. Overweight, obesity, and depression: a systematic review and metaanalysis of longitudinal studies. Arch Gen Psychiatry. (2010) 67:220-9. doi: 10.1001/archgenpsychiatry.2010.2

16. Penninx BW, Beekman AT, Honig A, Deeg DJ, Schoevers RA, van Eijk JT, et al. Depression and cardiac mortality: results from a communitybased longitudinal study. Arch Gen Psychiatry. (2001) 58:221-7. doi: 10.1001/archpsyc.58.3.221

17. Weinstein AA, Kallman Price J, Stepanova M, Poms LW, Fang Y, Moon J, et al. Depression in patients with nonalcoholic fatty liver disease and chronic viral hepatitis B and C. Psychosomatics. (2011) 52:127-32. doi: 10.1016/j.psym.2010.12.019

18. Ma Q, Yang F, Ma B, Jing W, Liu J, Guo M, et al. Prevalence of nonalcoholic fatty liver disease in mental disorder inpatients in China: an observational study. Hepatol Int. (2021) 15:127-36. doi: 10.1007/s12072-020-10132-z

19. Lee K, Otgonsuren M, Younoszai Z, Mir HM, Younossi ZM. Association of chronic liver disease with depression: a population-based study. Psychosomatics. (2013) 54:52-9. doi: 10.1016/j.psym.2012.09.005

20. Surdea-Blaga T, Dumitraşcu DL. Depression and anxiety in nonalcoholic steatohepatitis: is there any association? Rom J Intern Med. (2011) 49:273-80.

21. Youssef NA, Abdelmalek MF, Binks M, Guy CD, Omenetti A, Smith AD, et al. Associations of depression, anxiety and antidepressants with histological severity of nonalcoholic fatty liver disease. Liver Int. (2013) 33:1062-70. doi: $10.1111 /$ liv.12165

22. Choi JM, Chung GE, Kang SJ, Kwak M-S, Yang JI, Park B, et al. Association between anxiety and depression and nonalcoholic fatty liver disease. Front Med. (2021) 7:585618. doi: 10.3389/fmed.2020.585618

23. Moher D, Liberati A, Tetzlaff J, Altman DG, Group P. Preferred reporting items for systematic reviews and meta-analyses: the PRISMA statement. PLoS Med. (2009) 6:e1000097. doi: 10.1371/journal.pmed.1000097

24. Ismail Z, Elbayoumi H, Fischer CE, Hogan DB, Millikin CP, Schweizer T, et al. Prevalence of depression in patients with mild cognitive impairment: a systematic review and meta-analysis. JAMA Psychiatry. (2017) 74:58-67. doi: 10.1001/jamapsychiatry.2016.3162

25. Schwarzer G, Chemaitelly H, Abu-Raddad LJ, Rücker G. Seriously misleading results using inverse of freeman-Tukey double arcsine transformation in meta-analysis of single proportions. Res Synth Methods. (2019) 10:476-83. doi: $10.1002 /$ jrsm. 1348

26. Clopper CJ, Pearson ES. The use of confidence or fiducial limits illustrated in the case of the binomial. Biometrika. (1934) 26:404-13. doi: 10.1093/biomet/26.4.404

27. Koo CH, Chang JHE, Syn NL, Wee IJY, Mathew R. Systematic review and meta-analysis on colorectal cancer findings on colonic evaluation after CT-confirmed acute diverticulitis. Dis Colon Rectum. (2020) 63:701-9. doi: 10.1097/DCR.0000000000001664

28. Katz D, Baptista J, Azen SP, Pike MC. Obtaining confidence intervals for the risk ratio in cohort studies. Biometrics. (1978) 34:469-74. doi: $10.2307 / 2530610$

29. DerSimonian R, Laird N. Meta-analysis in clinical trials. Control Clin Trials. (1986) 7:177-88. doi: 10.1016/0197-2456(86)90046-2

30. Harris R, Bradburn M, Deeks J, Harbord R, Altman D, Sterne J. Metan: fixed- and random-effects meta-analysis. Stata J. (2008) 8:3-28. doi: $10.1177 / 1536867$ X0800800102

31. Fletcher J. What is heterogeneity and is it important? BMJ. (2007) 334:94-6. doi: 10.1136/bmj.39057.406644.68

32. Sedgwick P. What is publication bias in a meta-analysis? BMJ. (2015) 351:h4419. doi: 10.1136/bmj.h4419
33. Hunter JP, Saratzis A, Sutton AJ, Boucher RH, Sayers RD, Bown MJ. In metaanalyses of proportion studies, funnel plots were found to be an inaccurate method of assessing publication bias. J Clin Epidemiol. (2014) 67:897-903. doi: 10.1016/j.jclinepi.2014.03.003

34. Munn Z, Moola S, Riitano D, Lisy K. The development of a critical appraisal tool for use in systematic reviews addressing questions of prevalence. Int $\mathrm{J}$ Health Policy Manag. (2014) 3:123-8. doi: 10.15171/ijhpm.2014.71

35. Tomeno W, Kawashima K, Yoneda M, Saito S, Ogawa Y, Honda Y, et al. Non-alcoholic fatty liver disease comorbid with major depressive disorder: the pathological features and poor therapeutic efficacy. J Gastroenterol Hepatol. (2015) 30:1009-14. doi: 10.1111/jgh.12897

36. Elwing JE, Lustman PJ, Wang HL, Clouse RE. Depression, anxiety, and nonalcoholic steatohepatitis. Psychosom Med. (2006) 68:563-9. doi: 10.1097/01.psy.0000221276.17823.df

37. Balp MM, Krieger N, Przybysz R, Way N, Cai J, Zappe D, et al. The burden of non-alcoholic steatohepatitis (NASH) among patients from Europe: a real-world patient-reported outcomes study. JHEP Rep. (2019) 1:154-61. doi: 10.1016/j.jhepr.2019.05.009

38. Jung JY, Park SK, Oh CM, Chung PW, Ryoo JH. Non-alcoholic fatty liver disease and its association with depression in Korean general population. $J$ Korean Med Sci. (2019) 34:e199. doi: 10.3346/jkms.2019.34.e199

39. Moretti R, Caruso P, Gazzin S. Non-alcoholic fatty liver disease and neurological defects. Ann Hepatol. (2019) 18:563-70. doi: 10.1016/j.aohep.2019.04.007

40. Fernández MIC, Borges-González SA, Stepanova M, Infante-Velázquez ME, Ruenes-Domech C, González-Suero SM, et al. Health-related quality of life in cuban patients with chronic liver disease: a real-world experience. Ann Hepatol. (2020) 22:100277. doi: 10.1016/j.aohep.2020.10.005

41. Sayiner M, Arshad T, Golabi P, Paik J, Farhat F, Younossi ZM. Extrahepatic manifestations and healthcare expenditures of non-alcoholic fatty liver disease in the medicare population. Hepatol Int. (2020) 14:556-66. doi: 10.1007/s12072-020-10038-w

42. Labenz C, Huber Y, Michel M, Nagel M, Galle PR, Kostev K, et al. Nonalcoholic fatty liver disease increases the risk of anxiety and depression. Hepatol Commun. (2020) 4:1293-301. doi: 10.1002/hep4.1541

43. Ng M, Fleming $\mathrm{T}$, Robinson $\mathrm{M}$, Thomson $\mathrm{B}$, Graetz $\mathrm{N}$, Margono $\mathrm{C}$, et al. Global, regional, and national prevalence of overweight and obesity in children and adults during 1980-2013: a systematic analysis for the global burden of disease study 2013. Lancet. (2014) 384:766-81. doi: 10.1016/S0140-6736(14)60460-8

44. Comino E, Silove D, Manicavasagar V, Harris E, Harris M. Agreement in symptoms of anxiety and depression between patients and GPs: the influence of ethnicity. Family Pract. (2001) 18:71-7. doi: 10.1093/fampra/18.1.71

45. Chan KL, Cathomas F, Russo SJ. Central and peripheral inflammation link metabolic syndrome and major depressive disorder. Physiology. (2019) 34:123-33. doi: 10.1152/physiol.00047.2018

46. Raison CL, Capuron L, Miller AH. Cytokines sing the blues: inflammation and the pathogenesis of depression. Trends Immunol. (2006) 27:24-31. doi: 10.1016/j.it.2005.11.006

47. Gehrke N, Schattenberg JM. Metabolic inflammation-a role for hepatic inflammatory pathways as drivers of comorbidities in nonalcoholic fatty liver disease? Gastroenterology. (2020) 158:1929-47.e1926. doi: 10.1053 /j.gastro.2020.02.020

48. Egede LE, Zheng D, Simpson K. Comorbid depression is associated with increased health care use and expenditures in individuals with diabetes. Diabetes Care. (2002) 25:464. doi: 10.2337/diacare.25.3.464

49. Simon GE, Ludman EJ, Linde JA, Operskalski BH, Ichikawa L, Rohde P, et al. Association between obesity and depression in middle-aged women. Gen Hosp Psychiatry. (2008) 30:32-39. doi: 10.1016/j.genhosppsych.2007. 09.001

50. Sharafi SE, Garmaroudi G, Ghafouri M, Bafghi SA, Ghafouri M, Tabesh MR, et al. Prevalence of anxiety and depression in patients with overweight and obesity. Obesity Med. (2020) 17:100169. doi: 10.1016/j.obmed.2019.100169

51. Moieni M, Irwin MR, Jevtic I, Olmstead R, Breen EC, Eisenberger NI. Sex differences in depressive and socioemotional responses to an inflammatory challenge: implications for sex differences in depression. Neuropsychopharmacology. (2015) 40:1709-716. doi: 10.1038/npp.2015.17 
52. Quattrocki E, Baird A, Yurgelun-Todd D. Biological aspects of the link between smoking and depression. Harv Rev Psychiatry. (2000) 8:99-110. doi: 10.1080/hrp_8.3.99

53. Goodwin RD, Lavoie KL, Lemeshow AR, Jenkins E, Brown ES, Fedoronko DA. Depression, anxiety, and COPD: the unexamined role of nicotine dependence. Nicotine Tob Res. (2012) 14:176-83. doi: 10.1093/ntr/ntr165

54. Fan JG, Kim SU, Wong VW. New trends on obesity and NAFLD in Asia. $J$ Hepatol. (2017) 67:862-73. doi: 10.1016/j.jhep.2017.06.003

55. Hassan AM, Mancano G, Kashofer K, Fröhlich EE, Matak A, Mayerhofer $\mathrm{R}$, et al. High-fat diet induces depression-like behaviour in mice associated with changes in microbiome, neuropeptide $\mathrm{Y}$, and brain metabolome. Nutr Neurosci. (2019) 22:877-93. doi: 10.1080/1028415X.2018.14 65713

56. Parletta N, Zarnowiecki D, Cho J, Wilson A, Bogomolova S, Villani A, et al. A mediterranean-style dietary intervention supplemented with fish oil improves diet quality and mental health in people with depression: a randomized controlled trial (HELFIMED). Nutr Neurosci. (2019) 22:474-87. doi: 10.1080/1028415X.2017.1411320

57. Diabetes Prevention Program Research Group, Knowler WC, Fowler SE, Hamman RF, Christophi CA, Hoffman HJ, et al. 10-year follow-up of diabetes incidence and weight loss in the diabetes prevention program outcomes study. Lancet. (2009) 374:1677-86. doi: 10.1016/S0140-6736(09)61457-4

58. Stevens VJ, Obarzanek E, Cook NR, Lee IM, Appel LJ, Smith West D, et al. Long-term weight loss and changes in blood pressure: results of the trials of hypertension prevention, phase II. Ann Intern Med. (2001) 134:1-11. doi: 10.7326/0003-4819-134-1-200101020-00007

59. Gafoor R, Booth HP, Gulliford MC. Antidepressant utilisation and incidence of weight gain during 10 years' follow-up: population based cohort study. BMJ. (2018) 361:k1951. doi: 10.1136/bmj.k1951

60. Caiazzo R, Lassailly G, Leteurtre E, Baud G, Verkindt H, Raverdy V, et al. Roux-en-Y gastric bypass versus adjustable gastric banding to reduce nonalcoholic fatty liver disease: a 5 -year controlled longitudinal study. Ann Surg. (2014) 260:893-8. doi: 10.1097/SLA.0000000000000945

61. Laursen TL, Hagemann CA, Wei C, Kazankov K, Thomsen KL, Knop FK, et al. Bariatric surgery in patients with non-alcoholic fatty liver disease from pathophysiology to clinical effects. World J Hepatol. (2019) 11:138-49. doi: 10.4254 /wjh.v11.i2.138

62. Lee Y, Doumouras AG, Yu J, Brar K, Banfield L, Gmora S, et al. Complete resolution of nonalcoholic fatty liver disease after bariatric surgery: a Systematic review and meta-analysis. Clin Gastroenterol Hepatol. (2019) 17:1040-60.e1011. doi: 10.1016/j.cgh.2018.10.017

63. Backman O, Stockeld D, Rasmussen F, Näslund E, Marsk R. Alcohol and substance abuse, depression and suicide attempts after Roux-en-Y gastric bypass surgery. Br J Surg. (2016) 103:1336-42. doi: 10.1002/bjs.10258

64. Engel SG, Adair CE, Hayas CL, Abraham S. Health-related quality of life and eating disorders: a review and update. Int J Eat Disord. (2009) 42:179-87. doi: 10.1002/eat.20602
65. Steffen KJ, Engel SG, Wonderlich JA, Pollert GA, Sondag C. Alcohol and other addictive disorders following bariatric surgery: prevalence, risk factors and possible etiologies. Eur Eat Disord Rev. (2015) 23:442-50. doi: 10.1002/erv.2399

66. Zhou X, Teng T, Zhang Y, Del Giovane C, Furukawa TA, Weisz JR, et al. Comparative efficacy and acceptability of antidepressants, psychotherapies, and their combination for acute treatment of children and adolescents with depressive disorder: a systematic review and network meta-analysis. Lancet Psychiatry. (2020) 7:581-601. doi: 10.1016/S2215-0366(20)30137-1

67. Borenstein M, Higgins JPT, Hedges LV, Rothstein HR. Basics of meta-analysis: i2 is not an absolute measure of heterogeneity. Res Synth Methods. (2017) 8:5-18. doi: $10.1002 / \mathrm{jrsm} .1230$

68. Rücker G, Schwarzer G, Carpenter JR, Schumacher M. Undue reliance on I(2) in assessing heterogeneity may mislead. BMC Med Res Methodol. (2008) 8:79. doi: 10.1186/1471-2288-8-79

69. Ye Q, Zou B, Yeo YH, Li J, Huang DQ, Wu Y, et al. Global prevalence, incidence, and outcomes of non-obese or lean non-alcoholic fatty liver disease: a systematic review and meta-analysis. Lancet Gastroenterol Hepatol. (2020) 5:739-52. doi: 10.1016/S2468-1253(20)30077-7

70. Huang DQ, Yeo YH, Tan E, Takahashi H, Yasuda S, Saruwatari J, et al. ALT levels for asians with metabolic diseases: a meta-analysis of 86 studies with individual patient data validation. Hepatol Commun. (2020) 4:1624-36. doi: $10.1002 /$ hep 4.1593

Conflict of Interest: AS: President of Sanyal Biotechnology and has stock options in Genfit, Akarna, Tiziana, Indalo, Durect and Galmed. He has served as a consultant to Astra Zeneca, Nitto Denko, Enyo, Ardelyx, Conatus, Nimbus, Amarin, Salix, Tobira, Takeda, Jannsen, Gilead, Terns, Birdrock, Merck, Valeant, Boehringer-Ingelheim, Lilly, Hemoshear, Zafgen, Novartis, Novo Nordisk, Pfizer, Exhalenz and Genfit. He has been an unpaid consultant to Intercept, Echosens, Immuron, Galectin, Fractyl, Syntlogic, Affimune, Chemomab, Zydus, Nordic Bioscience, Albireo, Prosciento, Surrozen and Bristol Myers Squibb. His institution has received grant support from Gilead, Salix, Tobira, Bristol Myers, Shire, Intercept, Merck, Astra Zeneca, Malinckrodt, Cumberland and Novartis. He receives royalties from Elsevier and UptoDate.

The remaining authors declare that the research was conducted in the absence of any commercial or financial relationships that could be construed as a potential conflict of interest.

Copyright (ङ 2021 Xiao, Lim, Ng, Tan, Lim, Ho, Tan, Sanyal and Muthiah. This is an open-access article distributed under the terms of the Creative Commons Attribution License (CC BY). The use, distribution or reproduction in other forums is permitted, provided the original author(s) and the copyright owner(s) are credited and that the original publication in this journal is cited, in accordance with accepted academic practice. No use, distribution or reproduction is permitted which does not comply with these terms. 